\title{
Multiple Organ Failure in Septic Patients
}

Elizabeth Bilevicius, Desanka Dragosavac, Sanja Dragosavac, Sebastião Araújo, Antonio L.E. Falcão and Renato G.G. Terzi

\author{
Department of Surgery, Intensive Care Unit \\ (ICU), UNICAMP, Campinas, SP, Brazil
}

\begin{abstract}
Multiple organ failure (MOF) is the main cause of death in ICUs, especially affecting septic patients. It is strongly related to number of systems with failure, type of system involved, risk factors such as age, previous chronic diseases, delayed or inadequate resuscitation, persistent infection, immune suppression, and others. The prognoses is worse for patients rather than in elective or emergency surgical patients. The objective of this article is to provide data from our university teaching hospital ICU related to the incidence of septic patients, the distribution of MOF, and distribution of failure among each of the organs. The mortality rate, relationship between mortality and age, and mortality and types of organs affected were evaluated. The main bacterial causes of sepsis were also identified. A retrospective evaluation was done of 249 patients admitted to the ICU in a 4 month period during 1999. Fifty four patients had sepsis diagnosed by ACCS/SCCM criteria. There were 37 men and 17 women; 24 medical and 30 postsurgical patients ( 9 after elective surgery and 21 emergency patients). APACHE II score was calculated on admission and MOF, measured for the first five days, was diagnosed using Marshall and Meakins criteria. The statistical method used was non-parametric Mann-Whitney test, p $<0.05$ was considered significant. The incidence of sepsis was recorded in 54/249 patients (22\%). Thirty of these 54 patients $(56 \%)$ died. Death occurred in 2 of 11 pateints with one organ failure (18\%), in 14/27 with 2 or 3 organ failures (52\%), and 14/16 with 4 or more organ failures $(88 \%)$. None of the three patients 15 to 20 years old died, 17/32 (55\%) pateints age 21-60 years, and $>61$ years $13 / 19(68 \%)$, died. There were 23 patients with positive bacterial culture. The most frequent bacteria found were: Pseudomonas aeruginosa (5), multiresistant Acinetobacter baumanii (3), Staphylococcus epidermidis (3), Enterobacter aerogenes (3), Klebsiella pneumoniae (2) and multiresistant Staphylococcus aureus (2). The mean value \pm SD of APACHE II (mortality risk) for survivors was $21 \pm 18$ and for non-survivors $42 \pm 26$ $(p<0.001)$. We conclude that MOF due to sepsis in an ICU is frequent, with high mortality related to the number of failing organs, age and high APACHE II.
\end{abstract}

Key Words: APACHE II, sepsis, multiple organ failure.

Sepsis is a clinical syndrome of systemic inflammatory response due to an infectious process with a presumed or known focus (by means of culture) [1, $2,4,8]$. Multiple Organ Failure (MOF) is considered the resulting process [2]. MOF can be due to an

Received on 03 March 2001; revised 15 April 2001.

Address for correspondence: Dr. Elizabeth Bilevicius. Rua Antonio Cezarino 1003, apto 53, Zip code: 13015-291 Campinas, São Paulo. Brazil. E-mail: bilevicius@yahoo.com

The Brazilian Journal of Infectious Diseases 2001;5(3):103-110 (C) 2001 by The Brazilian Journal of Infectious Diseases and Contexto Publishing. All rights reserved. $1413-8670$ overwhelming inflammatory response secondary to trauma, ischemia or unclear systemic inflammation [7]. MOF is the main cause of death in Intensive Care Units (ICU), especially affecting septic patients $[1,3,4,7]$. Although supportive assistance to critically ill patients has improved a great deal, the mortality rates have remained the same in the last 2 decades $[3,8]$. These rates are directly related to factors such as number of organs affected $[3,6]$ and the different sources of involved systems [4, 7]. Unfortunately, conflicting results are seen in studies which try to correlate the different systems. This failure is attributed, by some authors, to the limited pathophysiologic knowledge of MOF. 
Risk factors such as age [4], previous chronic disease, inadequate/delayed resuscitation, and persistent inflammatory/infectious focus, increase the mortality and morbidity rates in septic patients $[3,4]$. A worse prognosis is seen in clinical patients than in elective or emergency surgical patients [2].

Although there is a great deal of research in this area related to different populations, it all points to the same evolutionary pattern of MOF. It seems that, once the process starts, it follows a general pattern of overwhelming response that can result in death in most cases.

There are no data related to incidence of sepsis, distribution of MOF, or distribution of failure that affects organs individually in our university teaching hospital ICU. It was our aim to provide these data in order to compare them with the literature. The mortality rate, relationship between mortality and age and mortality and types of organs individually affected were evaluated. The main bacterial populations were also identified.

\section{Materials and Methods}

This was a retrospective study of 249 patients admitted to our hospital ICU. They were admitted in a 4-month period during 1999. Fifty four patients were selected based upon ACCS/SCCM criteria described below [7] (at least 2 of the following must be present): temperature $>38^{\circ} \mathrm{C}$ or $<36^{\circ} \mathrm{C}$; heart rate $>90$ beats/ min; respiratory rate $>20$ breaths/min; white blood cell count $>12,000$ cells $/ \mathrm{mm}^{3},<4000$ cells $/ \mathrm{mm}^{3}$ or $>10 \%$ immature cells, and identified infection focus or positive culture. Diagnoses of the septic patients were: peritonitis 9 , pancreatitis 4 , pneumonia 10 , politrauma 11 , urinary infection 1, burns 1, ischemic stroke 2, StevensJohnson syndrome 1, cardiac surgery 7 , gastrointestinal surgery 3 , upper gastrointestinal bleeding 2, vascular surgery 1 , cholangitis 1 , and thoracic surgery 1 patient.

There were 37 men and 17 women; 24 clinical and 30 surgical patients ( 9 elective and 21 emergency surgical patients). APACHE II (mortality risk) was calculated on admission and MOF, measured for the first 5 days diagnosed by Marshall and Meakins criteria (Table 1). The statistical method used was non-parametric MannWhitney test, $\mathrm{p}$ value $<0.05$ was considered significant.

\section{Results}

The frequency of sepsis in our ICU was $22 \%$ and mortality rate among septic patients was $56 \%$ (30 of 54 patients). The number of system failures and mortality are seen in Table 2. Types of systems in failure and mortality are seen in Table 3. Age and mortality are seen in Table 4. Multiple organ failure affected more systems in non-survivors than in survivors; the difference between them reached statistical significance each day (Table 5).

The main bacterial populations found from 23 positive cultures (43\% of the patients had at least 1 positive culture) were: Pseudomonas aeruginosa (5 cultures), multiresistant Acinetobacter baumanii (3 cultures), Staphylococcus epidermidis (3 cultures), Enterobacter aerogenes (3 cultures), Klebsiella pneumoniae (2 cultures), multiresistant Staphylococcus aureus (2 cultures). The mean value \pm SD of APACHE II for survivors was $21 \pm 18$ and for non-survivors $42 \pm 26$ ( $p<0.001$ ), the Mann-Whitney test had a value of -3.20 .

\section{Discussion}

Although support for critically ill patients has significantly improved during the past 50 years, and knowledge about pathophysiology of conditions such as shock, acute renal failure, and acute respiratory failure has also improved, patients have longer survival, but mortality remains high. Patients started dying due to complications of their diseases, rather than the diseases themselves $[8,9]$. For the first time, physicians faced an overwhelming inflammatory response, leading to a progressive deterioration of patients'organ function with mortality rates up to $50 \%$ [2,9]. Actually, MOF became the main cause of death in ICUs, and, since the first studies which described this entity during the 1970s, mortality remains almost the same, in spite of all the research in laboratories and ICUs [2, 4, 6, 8].

The mortality of ICU septic patients ranges from $20 \%$ to $60 \%[4,6,9,19]$. Poole, et al. [8] observed a $66 \%$ mortality, while in our study it was $56 \%$. Mortality related to failure of 1 system was $18 \%$; 2 or 3 systems $52 \%$; 4 or more systems $88 \%$. Our study presented high mortality rates correlated to the number of systems affected. 
Figure 1. Mortality related to the number of systems with failure (MOF)

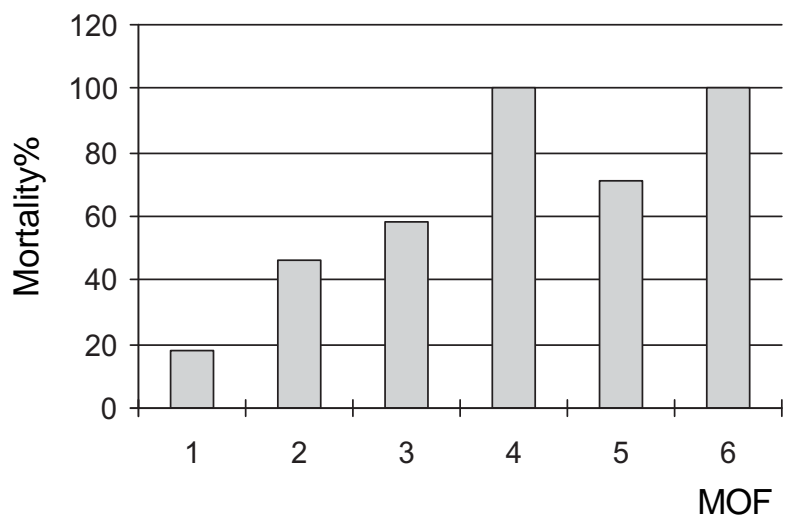

Table 1. Marshall and Meakins criteria for organ dysfunction

\begin{tabular}{ll}
\hline System/Organ & Dysfunction \\
\hline $\begin{array}{l}\text { 1. Respiratory } \\
\text { 2. Kidney }\end{array}$ & $\begin{array}{l}\text { lung injury score } \geq 1 \\
\text { serum creatinine }>1.8 \mathrm{mg} / \mathrm{dL}(160 \mathrm{umol} / \mathrm{L}) \\
\text { 3. Hepatic }\end{array}$ \\
$\begin{array}{l}\text { total bilirubin }>2.5 \mathrm{mg} / \mathrm{dL}(40 \mathrm{umol} / \mathrm{L}) \text { and elevation of transaminase } \\
\text { or alkaline phosphatase more than } 2 \text { times normal } \\
\text { PCWP }>16 \mathrm{mmHg} \text { and requirement for dopamine, dobutamine, } \\
\text { epinephrine and/or norepinephrine to maintain pressure }>80 \mathrm{mmHg}\end{array}$ \\
$\begin{array}{l}\text { Glagow coma scale }<10 \text { in the abscence of sedation } \\
\text { platelet count }<60,000 \text { and elevation of the prothrombin or partial } \\
\text { 6. Coagulation }\end{array}$ & $\begin{array}{l}\text { thromboplastin time }>1.5 \text { times the control value in absence of } \\
\text { anticoagulation } \\
\text { insulin requirements }>5 \mathrm{U} / \mathrm{h} \\
\text { nasogastric drainage }>300 \mathrm{~mL} / \mathrm{d} \text { and an ileus (not due to gut surgery) } \\
\text { upper gut bleeding }\end{array}$ \\
7. Metabolic &
\end{tabular}

Table 2. Number of systems in MOF and mortality

\begin{tabular}{cccc}
\hline MOF & $\mathbf{N}^{\circ}$ patients & Died & Mortality $\%$ \\
\hline 1 & 11 & 2 & 18 \\
$2-3$ & 27 & 14 & 52 \\
$>4$ & 16 & 14 & 88 \\
\hline Total & $\mathbf{5 4}$ & $\mathbf{3 0}$ & $\mathbf{5 6}$ \\
\hline
\end{tabular}


Figure 2. Mortality (\%) related to the type of system failure

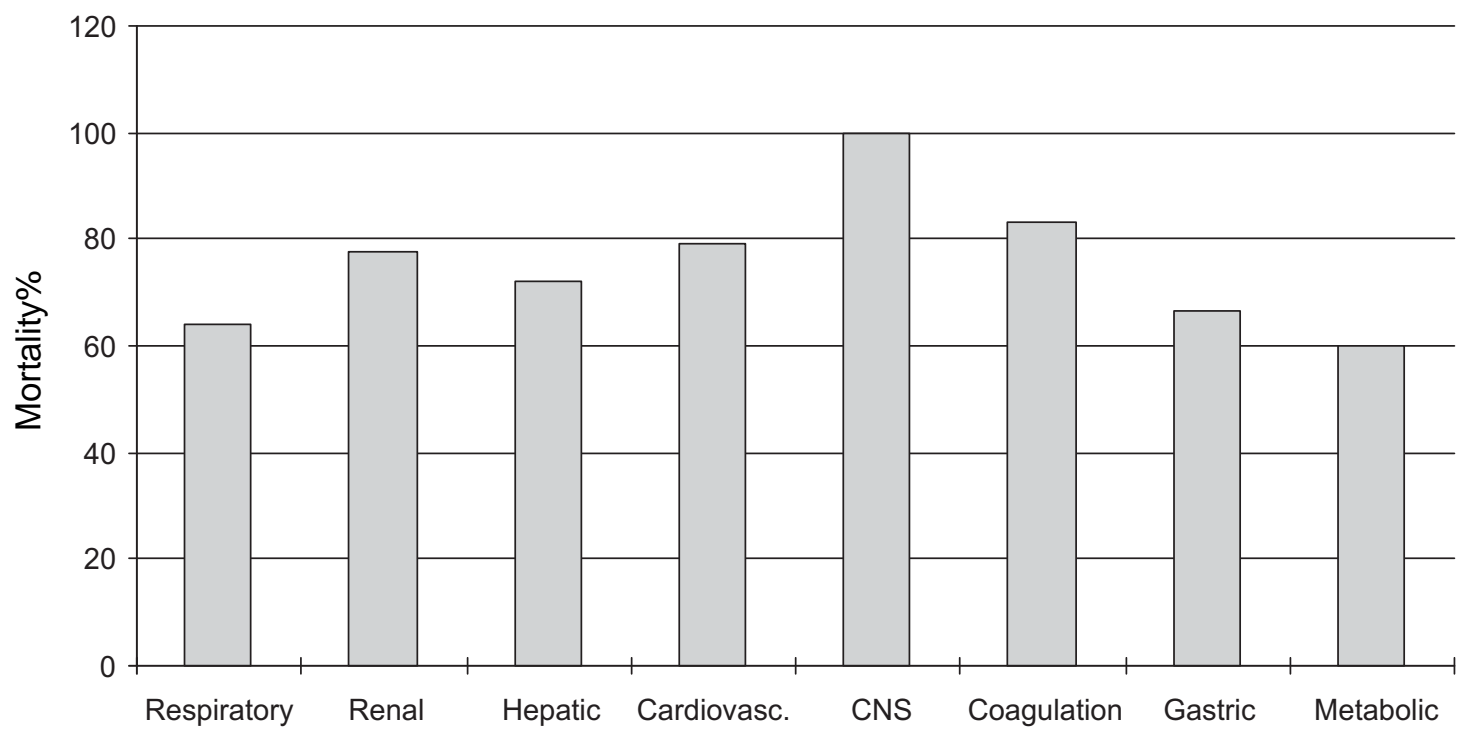

Table 3. Types of systems affected and mortality

\begin{tabular}{lccc}
\hline System failure & $\begin{array}{c}\mathbf{N}^{\circ} \text { patients with } \\
\text { each organ type } \\
(\mathbf{n}=\mathbf{5 4})\end{array}$ & Died & Mortality \% \\
\hline Respiratory & 51 & $33 / 51$ & 64 \\
Renal & 27 & $21 / 27$ & 78 \\
Hepatic & 18 & $13 / 18$ & 72 \\
Cardiovascular & 38 & $30 / 38$ & 79 \\
CNS & 3 & $3 / 3$ & 100 \\
Coagulation & 12 & $10 / 12$ & 83 \\
Gastrointestinal & 12 & $8 / 12$ & 67 \\
Metabolic & 5 & $3 / 5$ & 60 \\
\hline
\end{tabular}

Table 4. Age and mortality

\begin{tabular}{cccc}
\hline Age & $\mathbf{N}^{\circ}$ patients & Died & Mortality $\%$ \\
\hline $15-20$ & 3 & 0 & 0 \\
$21-60$ & 32 & 17 & 55 \\
$>61$ & 19 & 13 & 68 \\
\hline Total & $\mathbf{5 4}$ & $\mathbf{3 0}$ & $\mathbf{5 6}$ \\
\hline
\end{tabular}


Figure 3. Mortality related to the age

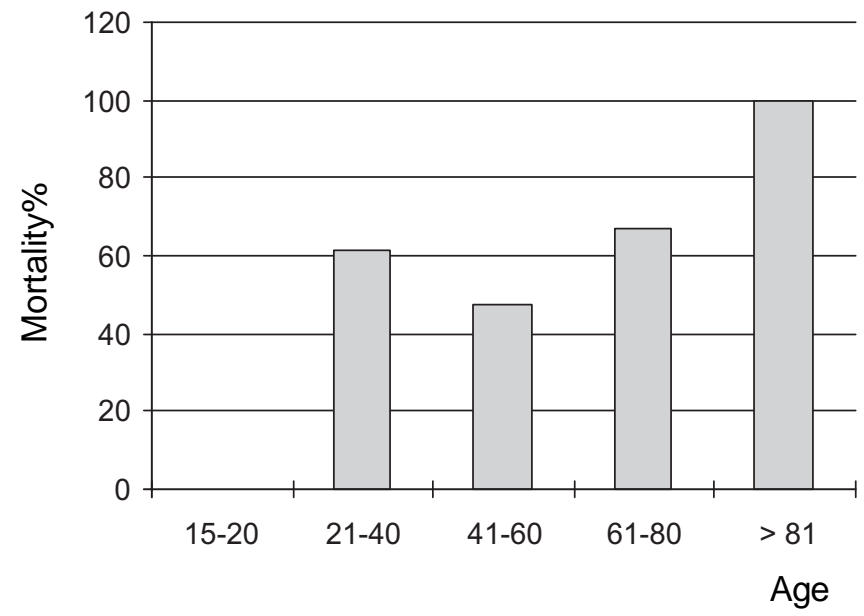

Table 5. Multiple organ failure in septic patients during the first 5 days of stay in the ICU

\begin{tabular}{llccc}
\hline Days & Group & Number of MOF $(\mathbf{M V}) *$ & SD & p-value \\
\hline MOF1 & survivors & 1.96 & 1.10 & \\
& non-survivors & 2.96 & 1.48 & 0.008 \\
MOF2 & survivors & 1.96 & 1.34 & \\
& non-survivors & 3.18 & 1.44 & 0.003 \\
MOF3 & survivors & 1.88 & 1.33 & \\
& non-survivors & 3.12 & 1.15 & 0.001 \\
MOF4 & survivors & 1.79 & 1.35 & \\
& non-survivors & 3.32 & 1.46 & 0.001 \\
MOF5 & survivors & 1.65 & 1.23 & \\
& non-survivors & 3.35 & 1.50 & 0.003 \\
\hline
\end{tabular}

* MV - mean value.

** SD - standard deviation.

Other studies show diversity of results with rates varying from $14 \%$ to $40 \%$ with 1 system failure; $20 \%$ to $76 \%$ with 2 system failures; $30 \%$ to $90 \%$ with 3 system failures [2, 3, 5, 6, 8, 9]; the majority of studies showed mortality of $100 \%$ for 4 or more system failures.

Hebert, et al. [3] found a strong linear correlation between the number of system failures and 30 day hospital mortality. Worse prognoses are seen in patients with MOF at onset of the sepsis syndrome. Mortality ranged from $10 \%$ for patients who had no organ system failure, to $100 \%$ in patients who had 5 or more organ system failures. The number of samples in this study related to 5 and 6 organ system failures was small and did not permit generalization, although we observed the same trend in whole in the literature (with larger samples) $[2,3,5,6,8,9,13]$. 
Age is an important comorbid factor, increasing the risk of death due to $\mathrm{MOF}(2)$. Worse prognoses are seen in patients who are older than 65 years $[2,3$, 13, 17]. Goris, et al. [6], did not find any clear relationship between age and MOF in their septic group, but they found a positive relationship between age and increased mortality in the trauma group. Our data show more than $55 \%$ mortality in all age groups older than 20 years. Based on our data, we could see that almost all patients (51/54) presented pulmonary failure, although we could not confirm that this was the very first system affected.

Gullo and Berlot [13] described a predictable and uniform clinical course of MOF. The first organ involved was lung, with almost all of their patients having this failure. Several other studies agree with their observation [6], relating lung dysfunction with high mortality $(>50 \%)$. These data are comparable with the mortality observed in ARDS alone. It could be explained by the fact that infection is the most common cause of ARDS [14]. It is probably due to the fact that lungs work as "first filters", activating cells such as neutrophils, lymphocytes, cytokines, lots of mediators leading to an increased capillary permeability, and fibrin-platelet aggregation due to activation of PAF and other septic mediators [14].

There is no consensus in the literature about the incidence and mortality of other system failures. This is probably due to the poor knowledge about sepsis and development of MOF, different parameters used for measuring insufficiency, and failure of systems and different follow up times for patients.

Cardiovascular system (CVS) failure is related to both high incidence and mortality $[3,6,12]$. In our study, 38/54 pateints had cardiovascular failure and 30 of them died. There are some doubts about when CVS failure appears. Regel, et al. [12], observed that CVS tends to fail late. Even with an abnormally high cardiac output associated with low systemic vascular resistance, clinical and biochemical alterations tend to occur in advanced phases only.

We observed a great number of patients who had renal failure (27/54). In other studies, however, we did not see the same trend. The kidney is a very important organ within the MOF context, probably predisposing to other failures. A 10-fold increase in mortality in patients with acute renal failure has been observed associated with other system failures [17]. Mortality due to acute renal failure alone decreased during the last 20 years. Acute renal failure secondary to sepsis, however, maintained the same levels (21 of 27 patients with renal failure in our study group died). During the past 20 years, the only observed change is that, now, the patients are older and with more underlying conditions. The mean total APACHE II has remained the same [18]. APACHE II was higher in non-survivors compared with survivors $(21 \%$ and $42 \%$, respectively).

Although we observed high mortality rates in coagulation failure (10/12), the frequency to this organ system failing was relatively low (12/54 patients). It is probable that this low frequency was artificial, in that we only classified patients with severe coagulopathy $\left(<60,000\right.$ platelets $\left./ \mathrm{mm}^{3}\right)$. What is observed during the MOF process is microscopic coagulopathy affecting only microvasculature, leading to a thrombotic process [11].

There are some indirect ways to diagnose disseminated intravascular coagulation (DIC) such as: acute renal failure, thrombosis and hemorrhage in the lungs, and liver failure [11]. Analysis of variables like fibrinogen concentration, factor II, factor V, platelet count, prothrombin time, and activated partial thromboplastin time, permits a diagnosis of DIC in more than $70 \%$ of septic patients $[10,19]$ with high mortality rates $[10,11,19]$. The parameter adopted was responsible for a low number of patients with $\mathrm{CNS}$ failure as well (3/54). We adopted the Glasgow coma scale score but, because a great number of ICU patients were sedated, we could not analyze them. This fact could explain the low incidence observed. High mortality is observed in other studies and incidence varies from $9 \%$ to $71 \%$ of septic patients [20].

We adopted an insulin requirement higher than $5 \mathrm{U}$ per hour as criteria for metabolic failure. Five of 54 septic patients presented this failure. The metabolic response during MOF is characterized as "auto cannabalism", a widespread catabolic response that 
alters carbohydrate, protein, fat, and mineral metabolism. This type of alteration leads to increased production of glucose with increased circulating glucose levels, accelerated proteolysis producing amino acids such as glutamine and alanine, which are the principal substrates for hepatic glucose production and accelerated lipolysis [21].

Another alteration (perhaps one of the first) is related to alterations in oxidative metabolism of mitochondria, that is considered a vital component in the evolution of sepsis [15]. There is an overwhelming production of free radicals (including NO), first by activated leukocytes, complement activation or initiation of ischemia-reperfusion mechanism, after this mitochondrion becomes the principal source of large amounts of free radicals [15]. There is also an increased amount of hormones stimulated by the infection, such as: epinephrine, norepinephrine, ACTH, cortisol, growth hormone, and glucagon. Insulin levels are often high, probably due to production of a very important mediator: TNF-alpha. This mediator is also responsible for induction of hyperglycemia [16]. Insulin level is a good parameter to measure metabolic failure. It reflects not only a primary stimulation, but also insulin resistance. At this stage, we observed severe MOF with high mortality rates $(60 \%)$.

In our study, we observed $42 \%$ (23/54) of patients with positive cultures. The most frequent bacteria were Pseudomonas aeruginosa, Enterobacter aerogenes, Acinetobacter baumanii, and Staphylococcus epidermidis. We analyzed APACHE II score (mortality risk) and it proved to be a good predictor of mortality and MOF: APACHE II in survivors was $21 \pm 18$ and in non-survivors $42 \pm 26$.

\section{Conclusion}

The frequency of sepsis in our ICU was $22 \%$ and mortality $56 \%$. We observed a correlation between mortality and number of systems with failure, as well as between mortality and age. There was significant difference in APACHE II values between survivors and non-survivors.

\section{References}

1. Nathens A.B., Marshall C. Sepsis, SIRS and MODS: What's in a name? World J Surg 1996;20:386-91.

2. Zimmerman J.E., Knaus W.A., et al. Severity stratification and outcome prediction for multisystem organ failure and dysfunction. World J Surg 1996;20:392-400.

3. Hebert P.C., Drummond A.J., Singer J., et al. A simple multiple system organ failure scoring system predicts mortality of patients who have sepsis syndrome. Chest 1993; 104:230-5.

4. Bossha K., Reijnders K., Hulstaert P.F., et al. Prognostic scoring system to predict outcome in peritonitis and intra-abdominal sepsis. Br J Surg 1997;84:1532-4.

5. Kollef M.H., Wragge T., Pasque C. Determinants of mortality and multiorgan dysfunction in cardiac surgery patients requiring prolonged mechanical ventilation. Chest 1995; 107:1395-401.

6. Goris R.J.A., te Boekhorst T.P.A., Nuytinck J.K.S., et al. Multiple organ failure: generalized autodestructive inflamation? Arch. Surg 1985;120:1109-15.

7. Bone R.C., Balk R.A., Cena F.B., et al. American College of Chest Physicians/ Society of Critical Care Medicine consensus conference definitions for sepsis and organ failure and guidelines for the use of innovative therapies in sepsis. Chest 1992;101:1644-55.

8. Poole G.V., Muakkassa F.F., Griswold J.A. The role of infection in outcome of multiple organ failure. Am Surg 1993;59:727-32.

9. Beal A.L., Cerra F.B. Multiple organ failure syndrome in the 1990s: systemic inflammatory response and organ dysfunction. JAMA 1994;271:226-33.

10. Thijs L.G., de Boer J.P., et al. Coagulation disorders in septic shock. Intensive Care Med 1993;19:S8-S15.

11. Baglin T. Disseminated intravascular coagulation: diagnosis and treatment. Br. J. Med 1996;312:683-7.

12. Regel G., Grotz M., Weltner T., et al. Pattern of organ failure following severe trauma. World J Surg 1996;20:460-4.

13. Gullo A., Berlot G. Ingredients of organ dysfuction or failure. World J Surg 1996;20:430-6.

14. Fulkerson W.J., MacIntyre W., Stamler J., et al. Pathogenesis and treatment of the adult respiratory distress syndrome. Arch Intern Med 1996;156:29-38.

15. Taylor D.E., Piantadosi C.A. Oxidative metabolism in sepsis and sepsis syndrome. J Crit Care 1995(3): 122-135.

16. Watters J.M., Wilmore D.W. Role of catabolic hormones in the hypoketonaemia of injury. Br J Surg 1986;73:108-10.

17. Levy E.M., Viscole C.M., Horwitz R.I. The effect of acute renal failure on mortality. JAMA 1996;275:1489-9.

18. McCarthy J.I. Prognosis of patients with acute renal failure in the intensive care unity: A tale of two eras. Mayo Clin Proc 1996; 71:117-26. 
19. Fourrier F., Chopin C., Goudemand J., et al. Septic shock, multiple organ failure and disseminated intravascular coagulation: compared patterns of antithrombine III, protein $\mathrm{C}$ and protein $\mathrm{S}$ deficiencies. Chest 1992;101:816-23.

20. Eidelman L.A., Putterman D., Putterman C., et al. The spectrum of septic encephalopathy. JAMA 1996; $275: 470-3$.

21. Mustard R.A., Bohnen J.M.A., Rosati C., et al. Pneumonia complicating abdominal sepsis: An independent risk factor for mortality. Arch Surg 1991;126:170-5. 